\title{
Prominence Eruptions
}

\author{
B. Vršnak \\ Hvar Observatory, Faculty of Geodesy, Kačićva 26, HR-10000 Zagreb, \\ Croatia
}

\begin{abstract}
The kinematics and dynamics of prominence eruptions are reviewed and different phases of the eruption are identified. The properties of the equation of motion in these phases are shown. The morphology of the prominence prior and during the eruption is described and the implications to the MHD description of the process are discussed.
\end{abstract}

\section{Introduction}

The evolution of prominences over their lifetime is characterized by several stages (Rompolt 1988) during which the internal structure gets more and more complex. When the structure becomes too intricate, the prominence becomes unstable and erupts (Rompolt 1990, Ballester 1994). The typical dimension of the system prior to the eruption is of the order of $10^{5} \mathrm{~km}$ but can vary from several $10^{4} \mathrm{~km}$ up to dimensions comparable to the solar radius. The time scale of an event is several hours, during which the prominence reaches the height in the range from several $10^{5} \mathrm{~km}$ up to 10 solar radii (Valniček 1968 , Tandberg-Hanssen et al. 1980, Athay and Illing 1986, Illing and Hundhausen 1986, Rompolt 1990). In the late phases of the eruption the prominence plasma becomes invisible in the $\mathrm{H} \alpha$ line either because of heating, or due to the decrease of the density caused by volume expansion or the mass loss through the legs of the prominence (Athay and Illing 1986, Illing and Hundhausen 1986, Vršnak et al. 1993). The velocity of the ejected prominence ranges from several tens of $\mathrm{km} / \mathrm{s}$ up to several hundereds of $\mathrm{km} / \mathrm{s}$ (Rompolt 1990). It is of the order of the intrinsic Alfvén velocity, revealing the magnetic nature of the process. Finally, let us stress that the 'frozen-in' condition is fulfilled (Vršnak 1992) so the morphology of the fine structure discloses the prominence's magnetic field skeleton and its evolution.

\section{Kinematics}

In Figure 1 the typical behaviour of an eruptive prominence is presented. The process of the eruption develops in several phases. In the pre-eruptive phase (denoted as phase 1 in Figure 1a) the prominence slowly rises with an approximately constant velocity in the order of $1-10 \mathrm{~km} / \mathrm{s}$. The internal structure gradually transforms from an intricate and chaotic structure into a simpler one, frequently characterized by helical-like patterns (Vršnak et al. 1991) sometimes also revealed by helical trajectories of the internal mass motions (Rušin and Ry- 


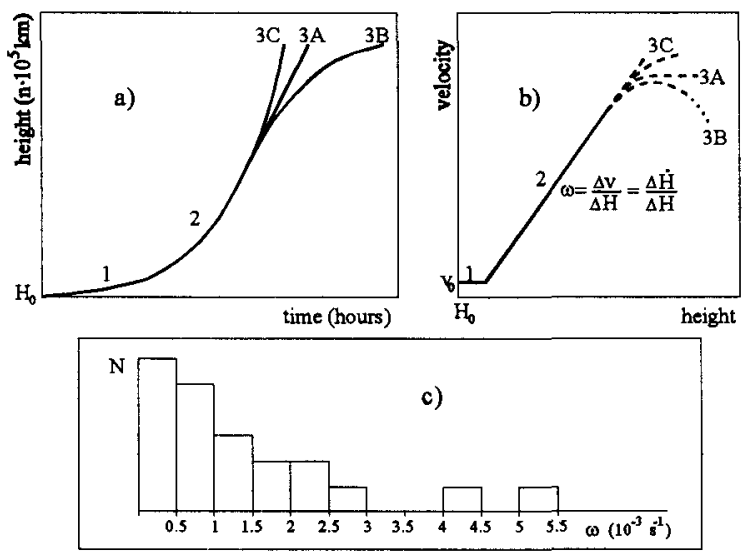

Figure 1. a) A typical dependence of the prominence height versus time. b) The graph (a) represented as the prominence velocity versus the height. c) The distribution of the growth rates $\omega=\dot{H} / H$ obtained from a sample of 20 prominences exposing $\dot{H} \propto H$ phase.

banský 1982). In the eruptions of the flare-spray type, the pre-eruptive phase is most often not observed (Tandberg-Hanssen 1974, Tandberg-Hanssen et al. 1980).

At some critical height (usually it is comparable with the prominence's footpoint half-distance), the prominence suddenly starts to accelerate (denoted as phase 2 in Figure 1a) and the velocity increases up to several hundreds of $\mathrm{km} / \mathrm{s}$. After the acceleration phase, the velocity often becomes constant (denoted as phase 3A in Figure 1a). Sometimes, the prominence continues to accelerate until it becomes invisible (phase $3 \mathrm{C}$ ) and sometimes a deceleration phase occurs (phase 3B). In one case it was observed that the prominence reached an upper equilibrium position and relaxed there as a damped oscillator (Vršnak et al. 1990).

In Figure $1 \mathrm{~b}$ the kinematics of the eruption is presented in the velocityheight graph $v(H)$, providing better insight into the dynamics of the process (Figure 2). The graph reveals that during the acceleration phase the velocity $v=\dot{H}$ is proportional to the height $H$. Out of 23 cases taken as illustrative samples, 20 prominences showed $\dot{H} \propto H$ phase with $\omega=\dot{H} / H \approx 10^{-3} \mathrm{~s}^{-1}$ (Figure 1c). In 9 cases the velocity reached $\dot{H}=$ const. phase (phase 3A), in 8 cases the velocity was continously increasing $(\dot{H} \propto H)$ untill the end of observations (phase $3 \mathrm{C}$ ), and in 3 cases the velocity was decreasing $\Delta \dot{H}<0$ (phase $3 \mathrm{~B}$ ). In the remaining three cases the phase 2 has not been identified. 

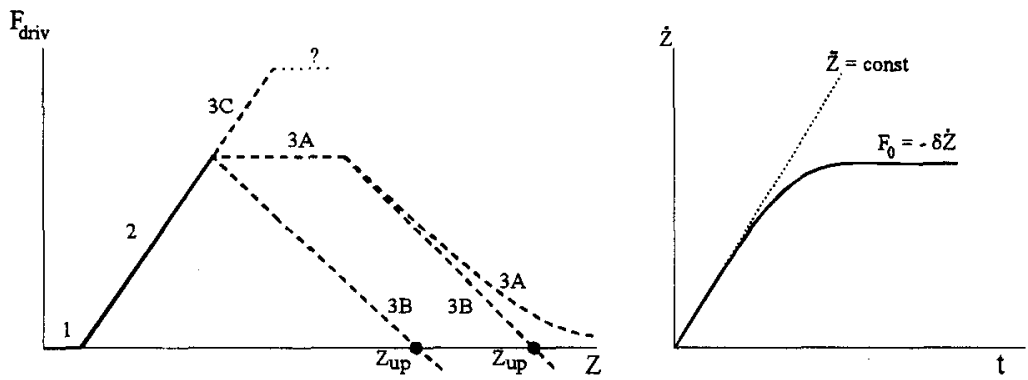

Figure 2. a) Properties of the driving force as inferred from the kinematics of the eruption. b) The behaviour of the velocity in the $F=$ const phase with the viscous drag of the form, $F_{v} \propto \dot{Z}$.

\section{Equation of Motion}

Figures $1 \mathrm{a}$ and $1 \mathrm{~b}$ reveal the basic properties of the equation of motion and the behaviour of the forces driving the eruption (Figure 2). Let us consider the equation of motion in the simplest form:

$$
\ddot{Z}=F(Z)-\delta \dot{Z} \text {. }
$$

Here, the dimensionless parameter $Z=H / D$ is introduced, i.e., the height is normalized with respect to the prominence's footpoint half-separation (Figure 3 ); the driving force (per unit mass) is denoted by $F(Z)$, and the term $\delta \dot{Z}$ represents the viscous drag (assumed to be proportional to the velocity). The value of the damping constant $\delta$ is of the order of $\delta \approx 10^{-3} \mathrm{~s}^{-1}$ as inferred from the observations of prominence oscillations (Ramsey and Smith 1966, Kleczek and Kuperus 1969, Tandberg-Hanssen 1974, Vršnak 1984, Vršnak et al. 1990, Vršnak 1993).

The pre-eruptive phase is characterized by $F(Z) \approx 0$ and $\delta \dot{Z} \approx 0$, so that the acceleration $\ddot{Z}=0$ (i.e., the velocity $\dot{Z}=$ const) and the prominence evolves through a series of equilibrium states. The second phase is characterized by $\dot{Z} \propto Z$. This implies that the viscous drag is still negligible and that $F(Z) \propto Z$ so that the equation of motion can be written in the form:

$$
\ddot{Z}=\omega^{2} Z,
$$

where we denoted $\omega=\Delta \dot{Z} / \Delta Z$ (Figure 1b). The solution of Eq. (2):

$$
Z \propto e^{\omega t},
$$

shows that the acceleration phase has the characteristics of a linear instability. The parameter, $\omega$, represents the growth rate of the instability and in the sample of 20 eruptive prominences having $\dot{Z} \propto Z$ it was most often close to the value of $10^{-3} \mathrm{~s}^{-1}$ (Figure 1c).

The phase $3 \mathrm{~A}$ characterized by $\dot{Z}=$ const can be comprehended in two ways. First, the driving force could be ceasing, as well as the viscous drag 


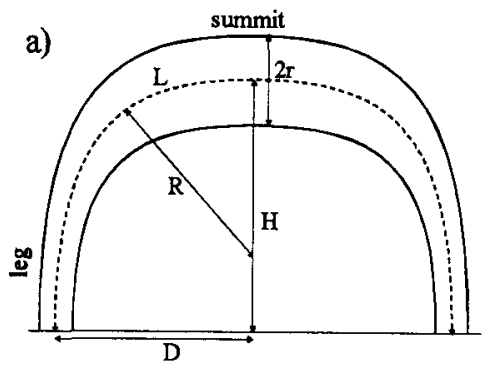

b)

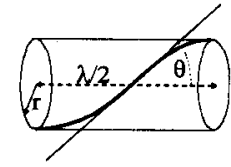

$$
\begin{aligned}
& X=\operatorname{tg} \theta=2 r \pi / \lambda \stackrel{? !}{=} \mathrm{B}_{\phi} / \mathrm{B}_{\eta \prime} \\
& \Phi=\mathrm{LX} / \mathrm{r} \quad \mathrm{N}=\Phi / 2 \pi
\end{aligned}
$$

Figure 3. a) Schematic drawing of an erupting prominence. b) $\mathrm{Pa}$ rameters defining a fine structure helical theread.

(due to the decrease of the coronal density), and so $\ddot{Z} \rightarrow 0$, meaning that the velocity becomes constant $(\dot{Z}=0)$. Another possibility is that $F(Z)$ becomes approximately constant and then, after a sufficiently high velocity is reached, the viscous drag balances the driving force (Figure 2b). Assuming that $F(Z)$ becomes constant $\left(F(Z)=F_{0}\right)$, the asymptotic solution of Eq. (1) gives $F_{0}=$ $\delta \dot{H}$. Taking $\delta=10^{-3} \mathrm{~s}^{-1}$ (Kleczek and Kuperus 1969, Vršnak et al. 1990) and the velocity of the value $v=\dot{H}=100 \mathrm{~km} / \mathrm{s}$ one obtains $F_{0}=100 \mathrm{~N} / \mathrm{kg}$. Taking for the density of the prominence $n=10^{16} \mathrm{~m}^{-3}$ one gets the force per unit volume of the order of $f_{0}=10^{-9} \mathrm{~N} / \mathrm{m}^{3}$, which is comparable with the 'magnetic forces', $f_{B}=B^{2} / 2 \mu_{0} R \approx 10^{-9}-10^{-8} \mathrm{~N} / \mathrm{m}^{3}$, proving that the eruption is of MHD origin. On the other hand, the deceleration of the prominence implies that the driving force has ceased and that the drag slows down the eruption.

Finally, there is a possibility that $F(Z)$ has such a shape that there exists an upper equilibrium position (denoted as $Z_{u p}$ Figure 2a). Then the prominence should relax at this position as a damped oscillator if $\omega_{u p}>\delta$ or should monotonically approach it if $\omega_{u p}<\delta$ (Vršnak et al. 1990)

\section{Morphology}

Eruptive prominences usually have a shape of an arch with legs anchored in the dense photosphere (Figure 3a). The parameters defining the arch are footpoint distance $(2 D)$, the height of the prominence axis summit $(H)$ the radius of the curvature $(R)$, the length of the axis $(L)$ and the prominence width $(2 r)$ which is usually larger at the summit than in the legs (Figure 3a). It is useful to normalize these parameters with respect to the footpoint half-distance, $D$, which is the only parameter constant during the process of eruption (e.g., $Z=H / D$, $l=L / D$, etc.). The internal structure frequently shows helical-like patterns (especially well exposed in the prominence legs) indicating that the prominence could be described in cylindrical geometry as a twisted magnetic flux tube of curved axis, with its footpoints anchored in the photosphere. In Figure $3 \mathrm{~b}$ the pitch angle, $\theta$, the pitch length, $\lambda$, the parameter, $X=\operatorname{tg} \theta$, the radius of the tube, $r$, as well as an effective twist, $\Phi$, are defined, and the parameter, $N$, represents the number of turns of a helical thread from one footpoint to the 
another. Taking into account that the 'frozen-in' condition is fulfilled (Vršnak 1992), one can relate the parameter, $X$, to the ratio of the azimuthal and the longitudinal component of the prominence magnetic field as $X=B_{\phi} / B_{\|}$.

In the pre-eruptive phase an initially intricate internal structure simplifies and in this stage the first signs of helical-like patterns appear (Rušin and Rybansky 1982, Vršnak et al. 1993). Usually the patterns are twisted more at the summit than in the legs (i.e., $X_{\text {summit }}>X_{\text {leg }}$ ) which reveals that the twist was transported into the expanded part of the tube (Jockers 1978, Browning and Priest 1983).

During the eruption, the ascending motion (causing streching of the prominence axis) is accompanied by the radial expansion of the tube (Ruždjak and Vršnak 1981) and the so-called 'detwisting' (Vršnak 1990a, Roša et al. 1993). This is not a proper term really, as the footpoints are anchored in the photosphere, so the total twist, $\Phi$, should remain constant if no internal reconnection of the magnetic field occurs. However, the parameter, $X$, decreases $\Delta X<$ 0 , implying that the stretching dominates over the radial expansion (Roša et al. 1993). The process of stretching of the helical patterns proceeds in such a way that the twist within an element of the tube $\left(\Phi^{\prime}\right)$ remains roughly constant (Vršnak 1990a, Vršnak et al. 1993, Roša et al. 1993), implying that the process of the transport of the twist into the expanding part of the prominence (at its summit) is too slow to be effective during the eruption. Sometimes, it is observed that the prominence axis screws, as expected from the screw mode instability (Sakurai 1976) implying that the internal twist is partly transported into the twist of the prominence axis. This can be significant when the dynamics of the eruption is considered, since it decreases the radius of the curvature at the summit of the prominence (Vršnak $1990 \mathrm{~b}, \mathrm{c}$ ).

The eruption is usually accompanied by mass loss (draining of the material downwards along the legs) which can be as large as $90 \%$ of the initial mass (Vršnak et al. 1993). The rate of mass loss is highest in the phase of acceleration, indicating that it is initiated by the inertial force at the prominence summit, and then controlled by gravity when draining along the prominence legs.

\section{Onset and Dynamics of the Eruption}

In an order of magnitude approach (Vršnak 1990b, c, Chen 1996) the forces per unit length, acting in the prominence and driving the eruption, could be represented in a rather simple form: $F_{m}=\mu_{0} I^{2} / 4 \pi H$ (the force due to the 'mirror current effect', Kuperus and Raadu 1974); $F_{k}=\mu_{0} I^{2} / 4 \pi R$ (the 'kink effect' term, Vršnak $1990 \mathrm{~b}, \mathrm{c}$ ); $F_{t}=-\mu_{0} I^{2} / 2 \pi R X^{2}$ (the 'tension effect' term, Vršnak b,c); $F_{g}=-m g$ (gravity); $F_{0}= \pm I B_{0}$ (the force due to an external field, Van Tend and Kuperus 1978); $F_{v}=-m \delta \dot{Z}$ (viscous drag). Here the current flowing along the axis of the prominence is denoted by $I$ and $m$ stands for the mass per unit length of the prominence $\left(m=r^{2} \pi \rho\right)$. Neglecting the dependence of the gravity force on the height and neglecting the viscous drag (approximation valid in the early stages of the eruption) the equation of motion can be written in the form:

$$
\ddot{Z}=A\left(\frac{L}{H}+\frac{L}{R}-\frac{2 L}{R X^{2}}\right),
$$




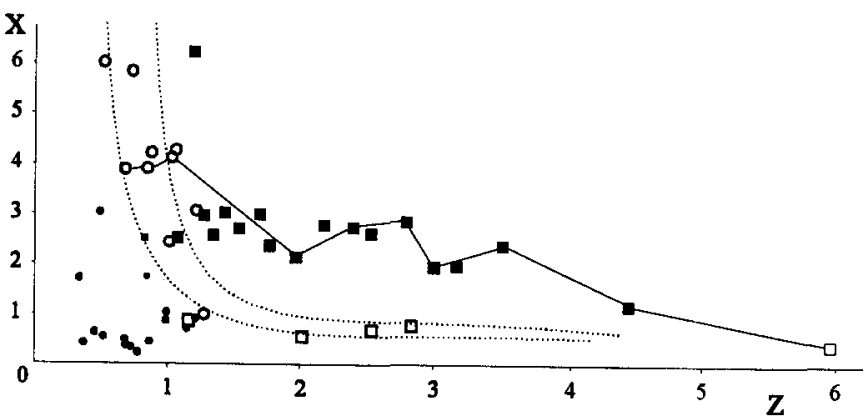

Figure 4. Stability diagram. Dotted lines represent the band in which the instability should occur. Dots represent the observed stable prominences, circles - the prominences at the onset of the eruption, black squares - prominences in the eruption, squares - the prominences in the post-acceleration phase. Full line represents the evolution of the eruptive prominence of August 18, 1980.

where:

$$
A=\frac{\mu_{0} I^{2}}{4 \pi M D}=\frac{B^{2}{ }_{\phi}}{\mu_{0} \rho L D} \approx \frac{v_{A}{ }^{2}}{L D} \approx \tau_{A}{ }^{-2} \approx \omega^{-2},
$$

Here $M$ is the total mass of the prominence $M=r^{2} \pi L \rho$, and $v_{A}$ and $\tau_{A}$ represent the Alfvén velocity and the Alfvén travel time along the axis of the prominence, respectively. Taking order of magnitude values for the magnetic field $B=10^{-3}$, $\mathrm{T}$ and $\rho=10^{-11} \mathrm{~kg} / \mathrm{m}^{3}$ for an average density, one finds the Alfvén velocity of the order of $100 \mathrm{~km} / \mathrm{s}$, i.e., the growth rate of the instability is of the order of $\omega \approx 10^{-3} \mathrm{~s}^{-3}$, consistent with the observations.

Linearizing Eq. (4) in the vicinity of the equilibrium position, and taking into account the complete set of $\mathrm{MHD}$ equations governing the behaviour of the parameter $I$ in various approximations (Vršnak 1990b, c, Chen 1996), one finds a set of curves, depending on the initial values of the relevant parameters $(I, \rho, r, L, D)$, which define the band in the graph $X(Z)$ where the instability should occur (denoted by the dotted lines in Figure 4). To the left of the indicated band prominences should be stable, and to the right they should be in the phase of acceleration. In Figure 4 the values of the parameter, $X$, measured close to the summit of the prominence axis in 28 cases, is presented versus the parameter, $Z$. One finds a good agreement (within the errors of the measurements) with the expectations based on Eq. (4) (Vršnak et al. 1991).

The scenario for the onset of the eruptive instability could be inferred from Eq. (4). An increase of the parameter, $A$, in the pre-eruptive phase (caused by $\Delta B_{\phi}>0$, i.e., $\Delta I>0$, or by $\Delta M<0$ ) shifts the equilibrium position to a larger height (Figure 5) and the whole function $\ddot{Z}(Z)$, given by Eq. (4), shifts upwards in the graph. The prominence is located at the height defined by the intersection of the curve $\ddot{Z}(Z)$ with the abcissa of the graph $(Z)$ characterized by $\Delta \ddot{Z}(Z) / \Delta Z<0$, meaning that for a small displacement from the equilibrium position the prominence should behave as an harmonic oscillator. For a larger displacement from the equilibrium (if it reaches the intersection characterized 


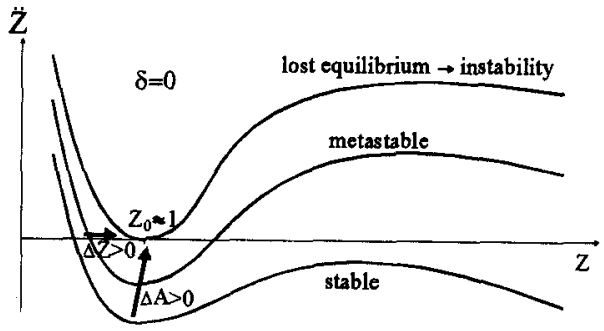

a)

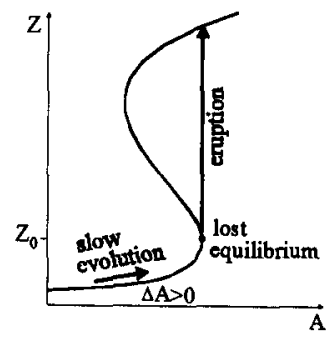

b)

Figure 5. a) Curves representing behaviour of the driving force as given by Eq. (4). b) Evolution of the prominence towards the eruption, caused by an increase of the parameter $A$.

by $\Delta \ddot{Z}(Z) / \Delta Z>0$ ) it should erupt: the prominence is in fact metastable. As the parameter, $A$, increases and the curve, $\ddot{Z}(Z)$, is shifted above the abscissa so that the values of the acceleration $\ddot{Z}$ are always positive, the prominence can not find a neighboring equilibrium position, i.e., it looses equilibrium and erupts (Figure 5b). The shape of the curves in Figure 5a determines the behaviour of the acceleration, and so the dynamics of the eruption. Taking into account the possible reconnection of the magnetic field lines below the prominence and the mass loss through the legs, one can get different variations of the curves shown in Figure 5a (Vršnak 1990b, c). However, basic features remain the same, and are in agreement with the behaviour of the driving force shown in Figure 2a. The solutions of the equation of motion allow also a monotonical ceasing of the acceleration, as well as the existence of an upper equilibrium position $\left(Z_{u p}\right)$ at which the prominence should relax as a damped oscillator due to the viscous drag (Vršnak 1990b).

\section{Discussion and Conclusions}

The kinematics of the eruption can be described by a rather simple equation of motion (Eq. (1)). The driving force is a result of several factors (Eq. 4). Substitution of Eq. (4) into Eq. (1) provides a comprehension of all basic features of the prominence eruption scenario. The agreement with the observations is good in the pre-eruptive phase, the onset of the eruption and the early stages of the eruption during which the prominence undergoes acceleration. Late phases of the eruption could be described in different ways, and the effects of mass loss and the reconnection of the magnetic field lines below the filament (like in the two-ribbon flare scenario) should be considered. The comprehension of the stability of prominences and transition to instability can be improved by analyzing their large scale oscillations (Vršnak 1993) in more detail. Finally, we stress that sometimes prominences relax to a lower energy state without eruption, i.e., by evolving through a series of equilibrium states (Vršnak and Ruždjak 1994).

Acknowledgments. I am grateful to students S. Korica and D. Nježić for help in preparation of $\dot{H}(H)$ graphs used for Figure 1b and 1c. 


\section{References}

Athay, R. G. and lling, R. M. E. 1986, J. Geophys. Res., 91, 10951

Ballester, J. L. 1994, in Solar Dynamic Phenomena and Solar Wind Consequences, ESA SP-373, p. 13

Browning, P. K., and Priest, E. R. 1983, ApJ, 266, 848

Chen, J. 1996, J. Geophys. Res., 101, 27499

Illing R. M. E. and Hundhausen, A. J. 1986, J. Geophys. Res., 91, 10951

Jockers, K. 1978, ApJ, 220, 1133

Kleczek, J. and Kuperus, M. 1969, Solar Phys., 6, 72

Kuperus M. and Raadu, M. A. 1974, A\&A, 31, 189

Ramsey, H. E., and Smith, S. F. 1966, AJ, 71, 197

Rompolt, B. 1988, in Dynamics and Structure of Solar Prominences, (eds.) J.

L. Ballester and E. R. Priest, Universitat de les Illes Ballears, p. 125

Rompolt, B. 1990, Hvar Obs. Bull., 14, 37

Roša, D., Vršnak B., Ruždjak, V., Ozguc, A. and Rušin, V. 1993, Hvar Obs. Bull., 17, 15

Rušin, V. and Rybanský, M. 1982, Bull. Astron. Inst. Czechosl., 33, 219

Ruždjak, V. and Vršnak, B. 1981, in Solar Maximum Year 2, (ed.) V. N. Obridko, Acad. of Sci. of Russia, Moscow, p. 256

Sakurai, T. 1976, Publ. Astron. Soc. Japan., 28, 177

Tandberg-Hanssen, E. 1974, Solar Prominences, D. Reidel, Dordrecht, Holland

Tandberg-Hanssen, E., Martin, S. F. and Hansen, R. T. 1980, Solar Phys., 65, 357

Valniček, B., 1968, in Structure and Development of Solar Active Regions, (ed.) K. O. Kipenheuer, D. Reidel, Dordrecht, Holland, p. 282

Van Tend, W., and Kuperus, M. 1978, Solar Phys., 59, 115

Vršnak, B. 1984, Solar Phys., 94, 289

Vršnak, B. 1990a, Solar Phys., 127, 129

Vršnak, B. 1990b, Solar Phys., 129, 295

Vršnak, B. 1990c, Astrophys. Space Sci., 170, 141

Vršnak, B. 1992, Ann. Geophys., 10, 344

Vršnak, B. 1993, Hvar Obs. Bull., 17, 23

Vršnak, B. and Ruždjak, V. 1994, Hvar Obs. Bull. 18, 1

Vršnak, B., Ruždjak, V., Brajša, R. and Zloch, F. 1990, Solar Phys., 127, 119

Vršnak, B., Ruždjak, V. and Rompolt, B. 1991, Solar Phys., 136, 151

Vršnak, B., Ruždjak, V., Rompolt, B., Roša, D. and Zlobec, P. 1993, Solar Phys., 146, 147 